\title{
Einleitung und Zielbestimmung
}

\section{Ausgangspunkt, Motivation und Zielbestimmung der Arbeit}

Dass berufliche Orientierung eine hohe individuelle und gesellschaftliche Relevanz besitzt, manifestiert sich nicht nur in der Weichenstellung für eine spätere Partizipation in der Arbeitswelt (Fend, 1991). Sie ist auch ein mögliches Vehikel zum Ausgleich von im Bildungssystem bestehenden Chancenungleichheiten (OECD, 2014).

Die berufliche Orientierung stellt für Jugendliche eine bedeutsame Entwicklungsaufgabe und große Herausforderung dar, die es neben anderen entwicklungsbezogenen Aufgaben in der Adoleszenz zu bewältigen gilt (Silbereisen, 1986). Während der Schulzeit sollen Heranwachsende ihre berufliche Identität entwickeln (Erikson, 1968) und berufswahlrelevante Kompetenzen erwerben, um den für ihre weitere berufliche Zukunft maßgeblichen nachschulischen Übergang vorzubereiten (Driesel-Lange, Kracke, Hany \& Kunz, 2020) und darüber hinaus ihren beruflichen Weg langfristig aktiv zu gestalten (Savickas, 2012).

Um dieser im Kontext beruflicher Entwicklung entstehenden Aufgabe in ihrer Komplexität gerecht werden zu können, benötigen Heranwachsende Unterstützung (Driesel-Lange et al., 2020). Dabei kommt der Institution Schule als substanziellem Handlungsfeld eine essenzielle Rolle zu. Zudem ist die Unterstützung des Berufswahlprozesses von Schüler*innen ein zentraler Bildungsauftrag der allgemeinbildenden weiterführenden Schulen in Deutschland (KMK, 2017b). Qua Gesetz teilen sie sich diese Aufgabe mit der Agentur für Arbeit ( $\$ 33$ SGB III). Neben diesen beiden Hauptakteurinnen existieren zahlreiche weitere Beteiligte wie Bundes- und Landesministerien, nachschulische Bildungsinstitutionen und Interessensverbände, die auf verschiedenen Handlungsebenen agieren. Ihr Verständnis beruflicher Orientierung differiert aufgrund unterschiedlicher sozial-, wirtschaftsund arbeitsmarktpolitischer, aber auch gesellschaftlicher und individueller Perspektiven und Ziele (Bührmann \& Wiethoff, 2013), die zusammengenommen 
einen komplexen kontextuellen (Handlungs-)Rahmen für die Umsetzung beruflicher Orientierung formen.

Die Komplexität beruflicher Orientierung erhöht sich darüber hinaus durch die Heterogenität der Jugendlichen. Aus der Heterogenität der Lernenden entstehen individuelle Bedarfe, die in einem iterativen Prozess bestehend aus Bedarfsdiagnose, Förderung und Evaluation adressiert werden sollten (Fischer, Rott, Veber, Fischer-Ontrup \& Gralla, 2014). Auch empirisch zeigt sich anhand der geringen statistischen Effekte ${ }^{1}$ nicht-individualisierter Maßnahmen (Whiston, Li, Goodrich Mitts \& Wright, 2017), dass ein entsprechender Bedarf existiert, berufsorientierende Unterstützung stärker an den individuellen Bedürfnissen auszurichten. Die Bedeutung der Ausrichtung beruflicher Orientierung an den individuellen Bedarfen, Interessen und Fähigkeiten der Heranwachsenden wird auch in den in der Konferenz der Kultusminister der Länder in der Bundesrepublik Deutschland (KMK) beschlossenen Zielen beruflicher Orientierung betont (KMK, 2017b).

Aufgrund personeller, organisatorischer und inhaltlich-didaktischer Herausforderungen, die durch fehlende Ressourcen, einen Informationsmangel hinsichtlich der Maßnahmeneffekte sowie das Fehlen systematischer Bedarfsanalysen entstehen (Ohlemann, Ittel, Rohowski \& Lazarides, 2016), ist die individuelle Förderung, im Sinne einer bedarfsorientierten Unterstützung, für Schulen bis dato nur schwer realisierbar (Rose \& Beutner, 2015). In der Konsequenz besuchen Schüler*innen eines Jahrgangs unabhängig von ihrem aktuellen Entwicklungsstand die gleiche berufsorientierende Maßnahme. Dabei stellt sich die Frage, welche Faktoren und Systematisierungen genutzt werden können, um die individuellen Bedarfe diagnostisch zu spezifizieren, zu gruppieren und schließlich auf Basis dieser Profilbildung eine Binnendifferenzierung beruflicher Orientierung im schulischen Kontext zu ermöglichen.

Ausgangspunkt dieser Arbeit ist insofern eine praxisorientierte Perspektive. Zum Verständnis der Multidimensionalität berufswahlbezogener Heterogenität der Jugendlichen soll eine systematische, theoriegeleitete Untersuchung der heterogenen Bedarfe aufgrund individueller Voraussetzungen, differierender Berufswahlstände sowie Unterschieden in der Entwicklung berufswahlrelevanter Kompetenzen vorgenommen werden. Des Weiteren soll eine empirische Grundlage geschaffen werden, um Schulen darin zu unterstützen, alle Jugendlichen entsprechend ihres individuellen Bedarfs in ihrer beruflichen Entwicklung bestmöglich zu fördern. Der

\footnotetext{
${ }^{1}$ Im Rahmen dieser Arbeit werden die Zusammenhänge zwischen individuellen Faktoren, berufsorientierenden Maßnahmen und der beruflichen Orientierung Jugendlicher untersucht. Mit Ausnahme der empirischen Studie in Kapitel 12 handelt es um querschnittliche Untersuchungen, über die keine Rückschlüsse auf kausale Effekte möglich sind. Es handelt sich also um statistische Effekte betrachteter Zusammenhänge.
} 
besondere Beitrag dieser Arbeit zur Untersuchung individueller Bedarfe von Schüler*innen in ihrer beruflichen Entwicklung liegt in der Identifikation demografischer, leistungs- und entwicklungsbezogener Faktoren und Muster. Die Ergebnisse dienen der Vertiefung der wissenschaftlichen Erkenntnis und der Sensibilisierung praxisrelevanter Akteur*innen hinsichtlich des Erfordernisses differenzierter, im Sinne bedarfsbezogener Planung, schulischer Berufs- und Studienorientierung. Schließlich bilden die empirischen Erkenntnisse dieser Arbeit Anknüpfungspunkte zur Weiterentwicklung bestehender Theorien.

Heterogenität wird in dieser Arbeit daher multidimensional betrachtet. Insbesondere stehen die Aspekte der Bedarfsdiagnostik, folglich das Verständnis der Unterschiede und ihrer Ursachen, sowie der Evaluation, also der Betrachtung von Wirkweisen berufsorientierender Maßnahmen in Abhängigkeit heterogener Bedingungen, im Fokus. Zur theoretischen Betrachtung lassen sich primäre und sekundäre Herkunftseffekte (vgl. Teilkapitel 3.2; Boudon, 1974), prestige- und geschlechterrollenbezogene Eingrenzungsprozesse (Gottfredson, 1981) sowie der milieugebundene Habitus (Bourdieu, 1982) heranziehen. Empirisch zeigt sich, dass Jugendliche mit Migrationshintergrund, aus sozial schwächeren und bzw. oder bildungsfernen Familien in Deutschland geringere Erfolgswahrscheinlichkeiten im Bildungssystem haben (Maaz, Neumann \& Baumert, 2014). Diese Chancenungleichheit lässt sich auch im Kontext beruflicher Entwicklung und im Laufbahnerfolg feststellen (Beicht \& Walden, 2019; Volmer \& Köppe, 2017). Dabei zeigen die wenigen existierenden, auf Intersektionalität ausgelegten Studien (Beicht \& Walden, 2019; Schmude, 2010), dass die Ausgangslage und die berufliche Entwicklung der Jugendlichen sowie ihre daraus resultierenden Bedarfe aufgrund intersektionaler Beziehungen zwischen den einzelnen Faktoren noch stärker differieren. Unterscheiden sich die Jugendlichen in ihren Bedarfen, so liegt die Schlussfolgerung nahe, dass auch die unterstützenden Maßnahmen heterogene Effekte aufweisen. Jedoch erfolgt die Evaluation berufsorientierender Maßnahmen ,eher unsystematisch“" und mit ,wenig zufriedenstellende[n] Ergebnisse[n]“ (S. 58), wie Driesel-Lange et al. (2020) kritisch feststellen. Eine systematische Untersuchung, wie die intersektionalen Zusammenhänge die Effekte berufsorientierender Maßnahmen beeinflussen, steht demzufolge noch aus. Dabei ist dieses Wissen zentral für die praktische Ausgestaltung bedarfsorientierter Unterstützung.

Auf der Grundlage des beschriebenen Desiderats leistet die vorliegende Arbeit einen besonderen Beitrag zur Untersuchung und Identifizierung individueller Unterstützungsbedarfe Jugendlicher, indem ihre berufliche Orientierung in Abhängigkeit der Teilnahme an verschiedenen berufsorientierenden Maßnahmen analysiert wird. Dabei wird ein Fokus auf die Effekte soziodemografischer und leistungsbezogener Merkmale der Schüler*innen gelegt. 
Ein weiterer Schwerpunkt der Arbeit liegt in der Analyse der Berufswahlkompetenzentwicklung Jugendlicher, denn neben soziodemografischen Merkmalen und der Schulleistung beeinflusst die Ausprägung von Kompetenzen zur Überwindung berufswahlbezogener Herausforderungen ihren weiteren Unterstützungsbedarf (Driesel-Lange et al., 2020). Das Berufswahlkompetenzmodell von Driesel-Lange, Hany, Kracke und Schindler (2010) definiert Berufswahlkompetenz als ein Konstrukt aus insgesamt zwölf Wissens-, Motivations- und Handlungsfacetten und liefert die theoretische Grundlage der empirischen Studien in Kapitel 10 bis 12. Obgleich bereits Instrumente zur Diagnostik individueller Entwicklungsstände vereinzelt existieren (Hirschi et al., 2019; Lipowski, Kaak, Kracke \& Holstein, 2015b) und Lehrkräfte diese in der Theorie auch kennen, finden Kompetenzdiagnostikinstrumente dennoch selten Anwendung (Beutner, Kremer \& Zoyke, 2012). Gründe könnten in den fehlenden zeitlichen Ressourcen (Ohlemann et al., 2016), der Komplexität der Anwendungsverfahren sowie in der mangelnden diagnostischen Kompetenz der Lehrkräfte (Schnebel, 2017) zu finden sein.

Es stellt sich also die Frage, inwiefern die Diagnostik des Entwicklungsstands der Jugendlichen im schulischen Kontext vereinfacht und dadurch realisierbar werden könnte. In Anlehnung an den mehrere Intelligenzbereiche zusammenfassenden Intelligenzquotienten (Bühner, 2011) könnte ein übergreifender Normwert auch die Diagnostik der Entwicklungsstände vereinfachen. Ein Beitrag dieser Arbeit zur Berufswahlkompetenzforschung liegt in der Untersuchung eines entsprechenden Normwerts der Berufswahlkompetenz als Ausgangspunkt schulischer Diagnostik. Mit Blick auf die eingangs angeführten Herausforderungen in der Praxis, scheint eine vollständig individualisierte Unterstützung beruflicher Orientierung, im Sinne eines Individualplans, derzeit nur schwer umsetzbar (Ohlemann et al., 2016; Rose \& Beutner, 2015). Unter Berücksichtigung dessen ist ein weiterer Leitgedanke dieser Arbeit, Jugendliche mit ähnlichen Bedürfnissen zu identifizieren und zu gruppieren, um für diese Kleingruppen, im Sinne einer Binnendifferenzierung, endsprechend maßgeschneiderte Anpassungen vorzunehmen und damit eine praxisnahe, realisierbare Lösung partieller Individualisierung im Lichte bestehender Umsetzungsrestriktionen zu ermöglichen (Bastian et al., 2016). Vor dem Hintergrund dieser Problematik werden zudem folgende Fragen untersucht:

Welche Berufswahlkompetenzprofile lassen sich bei den Jugendlichen identifizieren?

Welche Ausprägungsmuster der einzelnen Berufswahlkompetenzfacetten bestehen?

Lassen sich im Berufswahlkompetenzerwerb der Jugendlichen verschiedene Bewegungsmuster abbilden? 
Zur Erfassung von Mustern im Berufswahlkompetenzstand von Jugendlichen existieren erste Studien (Driesel-Lange \& Kracke, 2017; Kaak, Heinrichs, Lipowski, Wuttke \& Kracke, 2015; Ohlemann \& Driesel-Lange, 2017), die jedoch alle auf einer variablenzentrierten Analysemethode basieren und eine querschnittliche Betrachtung vornehmen. Indem der Berufswahlkompetenzstand von Schüler*innen sowie deren Entwicklung über die Zeit anhand eines personenzentrierten Ansatzes als latente Berufswahlkompetenzprofile und latente Entwicklungsprofile sichtbar gemacht werden, leistet diese Arbeit einen wichtigen Beitrag zur Befundlage des Erwerbs von Berufswahlkompetenz. Die zeitlichen Verlaufsmuster lassen zudem Rückschlüsse für die Theoriebildung zu, insbesondere in Hinblick auf die Diskontinuität im beruflichen Entwicklungsprozess. Die vorgestellte Arbeit verfolgt darüber hinaus das Ziel, die schulische Praxis, im Sinne des durch Lent (2017) geforderten Theorie-Empirie-Praxistransfers, in der Umsetzung binnendifferenzierter beruflicher Orientierung zu unterstützen.

\section{Gliederung der Arbeit}

Die folgenden Kapitel gliedern sich in einen theoretischen und einen empirischen Teil sowie eine Gesamtdiskussion. Kapitel 2 bis 7 bilden zusammen den theoretischen Teil. In ihm werden die zentralen Begriffe dieser Arbeit entfaltet. Des Weiteren werden die theoretischen Konstrukte und Modelle, auf denen die empirischen Studien des zweiten Teils basieren, beschrieben und mit der aktuellen empirischen Befundlage verschränkt. Die Kapitel 8 bis 12 formen den empirischen Teil. Nach der Darstellung der Datenlage sowie der angewandten statistischen Methoden werden die Ergebnisse der vier empirischen Studien vorgestellt. In Kapitel 13 werden die Ergebnisse des empirischen Teils im Rahmen einer Gesamtdiskussion zusammengefasst, im Kontext der Theorie sowie der empirischen Befundlage interpretiert und in diese eingeordnet. Im Folgenden wird der Aufbau der Arbeit nochmals im Detail entfaltet.

Aufgrund der Verortung berufsorientierender Unterstützung von Jugendlichen an und in der Institution Schule, wird in Kapitel 2 zunächst das Spannungsfeld der schulischen Berufs- und Studienorientierung mit ihren Zielen, beteiligten Akteur*innen und ihren differierenden Perspektiven dargestellt. Dabei zeigt sich die Bedeutung der von der KMK im Jahr 2017 verabschiedeten Erklärung zur einheitlichen Verwendung des Begriffs der beruflichen Orientierung sowie den anzustrebenden Zielen eben dieser. Zum anderen wird deutlich, dass für Schulen eine große Herausforderung in der erfolgreichen Umsetzung beruflicher Orientierung im Kontext der Heterogenität ihrer Schüler*innen und der notwendigen Adressierung ihrer entsprechend heterogenen Bedarfe besteht. 
Daher werden in Kapitel 3 - nach einer Definition und begrifflichen Einordnung von Heterogenität - drei Theorien vorgestellt, die zur Erklärung bestehender Unterschiede im Berufswahlverhalten der Jugendlichen anhand ihrer persönlichen Merkmale und Voraussetzungen herangezogen werden können. Die im gleichen Kapitel vorgestellte empirische Befundlage beleuchtet zudem den bestehenden Forschungsbedarf zu den intersektionalen Effekten dieser in der beruflichen Orientierung bisher vor allem einzeln betrachteten Merkmale. Diese Forschungslücke adressiert die empirische Studie in Kapitel 9. Bei der Spezifizierung individueller Bedarfe müssen neben den unterschiedlichen Ausgangslagen der Heranwachsenden auch die verschiedenen Phasen, in die sich die berufliche Orientierung gliedern lässt, berücksichtigt werden. In Kapitel 4 werden ausgewählte Modelle differenzierter beruflicher Entwicklung dargelegt. Es zeigen sich berufswahlbezogene Herausforderungen, die sich als phasentypisch darstellen. Die zur Bewältigung dieser berufswahlbezogenen Herausforderungen benötigten Kompetenzen werden in Kapitel 5 genauer betrachtet und ausgewählte theoretische Modelle berufswahlrelevanter Kompetenzen und Ressourcen vorgestellt. Es zeigt sich, dass zur Erfassung von individuellen Bedarfen eine theoretische Verschränkung der individuellen Voraussetzungen mit dem Berufswahlprozess und den berufswahlrelevanten Kompetenzen benötigt wird. Das Kapitel 6 ist folglich der Beschreibung des Thüringer Berufswahlkompetenzmodells gewidmet, das diese Verschränkung und zudem eine Einbettung in den schulischen Kontext vornimmt. Nach einer Vorstellung des theoretischen Modells und seiner empirischen Operationalisierung wird in einer Gegenüberstellung mit den zuvor präsentierten Modellen seine Auswahl als theoretische Grundlage der empirischen Studien in Kapitel 10 bis 12 begründet.

Ausgehend von den vorgestellten theoretischen und empirischen Befunden werden in Kapitel 7 die Ziele und die leitende Forschungsfrage dieser Arbeit formuliert und in die Forschungsfragen der enthaltenen Studien übergeleitet.

Der empirische Teil dieser Arbeit besteht aus vier Studien. Ihnen vorangestellt ist die Beschreibung des methodischen Vorgehens in Kapitel 8. Es werden die wissenschaftlichen Projekte, aus denen die Stichproben der einzelnen Studien stammen, präsentiert und die statistischen Verfahren erläutert sowie ihre Wahl in Abgrenzung $\mathrm{zu}$ anderen methodischen Zugängen begründet. Die in Kapitel 9 vorgestellte Studie greift das Forschungsdesiderat der intersektionalen Betrachtung individueller Merkmale Jugendlicher bei der Bewertung berufsorientierender Maßnahmen und ihrer differierenden Effekte auf die berufliche Orientierung auf. Die empirische Studie in Kapitel 10 befasst sich mit der für Schulen aufgrund ihrer Komplexität schwierigen Diagnostik berufswahlbezogener Entwicklungsstände der Schüler*innen. Zur Komplexitätsreduzierung der Diagnostik des individuellen Berufswahlkompetenzstands wird die Formung eines Normwerts der Berufswahlkompetenz als 
methodische Lösung vorgestellt und erörtert. Ein zentraler Aspekt ist dabei die Analyse, inwiefern ein zentraler Normwert die individuelle Ausprägung der einzelnen Berufswahlkompetenzfacetten gemäß dem Thüringer Modell wiedergeben kann.

Die in Kapitel 11 beschriebene empirische Studie erweitert die vorangegangenen Analysen der Ursachen heterogener Bedarfe und der Normierung des Berufswahlkompetenzstands um den Aspekt der Profilbildung, der als Ausgangspunkt binnendifferenzierter Unterstützung beruflicher Orientierung dienen kann.

Die empirische Studie in Kapitel 12 ergänzt diese Erkenntnisse um eine längsschnittliche Perspektive auf den Berufswahlkompetenzstand Jugendlicher und ermöglicht entsprechend Rückschlüsse auf die Entwicklungsverläufe in der Adoleszenz. Dabei werden die latenten Bewegungsmuster der Schüler*innen zwischen den zuvor identifizierten Berufswahlkompetenzprofilen untersucht, um Aufschluss über die individuellen Entwicklungsmuster als Quelle individueller Bedarfe einerseits und als Anknüpfungspunkt zur schulinternen Binnendifferenzierung andererseits zu gewinnen. In Kapitel 13 werden in einer Gesamtdiskussion zunächst die gewonnenen Erkenntnisse zusammengefasst und dann unter Einbezug des gegenwärtigen empirischen Forschungsstands interpretiert und eingeordnet. Zur kritischen Würdigung der vorliegenden Arbeit werden ihre Grenzen und die sich daraus für zukünftige Forschungsarbeiten ergebenden Herausforderungen dargelegt. Die aus dieser Arbeit neu entstandenen Forschungsdesiderata werden in Konsequenzen für die weitere wissenschaftliche Arbeit übersetzt. Abschließend werden die Implikationen der vorgestellten Ergebnisse für die schulpädagogische Praxis im Konkreten und den erweiterten Kreis der Akteur*innen beruflicher Orientierung im Allgemeinen diskutiert. Die Arbeit schließt mit einer zusammenfassenden Conclusio in Kapitel 14. 
Open Access Dieses Kapitel wird unter der Creative Commons Namensnennung 4.0 International Lizenz (http://creativecommons.org/licenses/by/4.0/deed.de) veröffentlicht, welche die Nutzung, Vervielfältigung, Bearbeitung, Verbreitung und Wiedergabe in jeglichem Medium und Format erlaubt, sofern Sie den/die ursprünglichen Autor(en) und die Quelle ordnungsgemäß nennen, einen Link zur Creative Commons Lizenz beifügen und angeben, ob Änderungen vorgenommen wurden.

Die in diesem Kapitel enthaltenen Bilder und sonstiges Drittmaterial unterliegen ebenfalls der genannten Creative Commons Lizenz, sofern sich aus der Abbildungslegende nichts anderes ergibt. Sofern das betreffende Material nicht unter der genannten Creative Commons Lizenz steht und die betreffende Handlung nicht nach gesetzlichen Vorschriften erlaubt ist, ist für die oben aufgeführten Weiterverwendungen des Materials die Einwilligung des jeweiligen Rechteinhabers einzuholen.

(c) (9) 